\title{
Plane polymer configurations enclosing a fixed area in an electric field: Generating functional and statistical mechanical properties
}

\author{
D.C. Khandekar ${ }^{1}$ and F.W. Wiegel \\ Center for Theoretical Physics, Twente University, P.O. Box 217, 7500 AE Enschede, \\ The Netherlands
}

Received 25 September 1992

\begin{abstract}
The statistical mechanical properties of plane polymer configurations which enclose a fixed area and are subject to an external electric field are investigated. For this purpose an exact expression for the generating functional is obtained and subsequently used to derive: (a) the distribution function for the enclosed area; (b) the mean square distance of a given repeating unit from the origin; (c) the entropic force on a repeating unit.
\end{abstract}

\section{Introduction}

Many properties of polymeric systems are due to the mutual entanglement of these chain-like objects. A model for the statistical mechanical properties of such systems was proposed for the first time by Edwards [1]. In this model a planar polymer was assumed to entangle with another one which was modelled as an infinite straight line perpendicular to the plane of the first polymer. The evaluation of the statistical mechanical properties involved the calculation of a path integral with a topological constraint. Since then the model has been extensively studied. For example, Khandekar and Wiegel [2] proposed an approach in which one studies the configurations on the associated covering space. A review of the mathematical description of various simple entanglements can be found in a recent monograph [3]. The various exactly solvable cases involving constraints are discussed by Khandekar and Lawande [4]. However, in all these cases the number of polymers which are entangled with each other was restricted to 2 . This shows the need for a model which can take into account the entanglement of clusters of polymers.

\footnotetext{
${ }^{1}$ Permanent address: Theoretical Physics Division, Bhabha Atomic Research Centre, Bombay 400085 , India.
} 
Brereton and Butler [5] suggested another model to study the mutual entanglement of many polymers. The essential ingredients of the model were: (1) represent a polymer by a discretc random walk of $N$ steps in a planc; (2) approximate the steric and topological effects of the other polymers in the system by the constraint that this walk encloses a fixed area. Because of the discrete nature of the model these authors had to resort to numerical methods for an explicit computation of the thermodynamic properties.

Khandekar and Wiegel [6] simplified the Brereton-Butler model further by assuming a continuous random walk to represent the polymer. They were able to map the problem of finding the configuration sum onto the evaluation of Levy's "stochastic area" [7] for a "Brownian bridge". Their results were later generalized by Duplantier [8], who calculated the distribution of the area in several complex cases. For these continuous walks Khandekar and Wiegel found the distribution of the area; they also studied the thermodynamic properties of these systems without any interaction between the monomers [9]. In a later publication [10] monomer-monomer interactions were also included.

However, if one wants to study the properties of DNA in gel electrophoresis, one needs to put the system in an external electrical field of constant magnitude and direction. The electric field is expected to alter the properties considerably. It is, therefore, the aim of this paper to study the thermodynamic properties of a plane polymer, enclosing a fixed area, subject to an external electrical field.

\section{Basic formulation}

The configuration sum or fraction of polymer configurations $G_{0}\left(r^{\prime \prime}, N \mid r^{\prime}, 0\right)$ of the non-interacting polymer, whose two ends are fixed at $r^{\prime}$ and $r^{\prime \prime}$ respectively, can be expressed as a Wiener integral

$$
G_{0}\left(\boldsymbol{r}^{\prime \prime}, N \mid \boldsymbol{r}^{\prime}, 0\right)=\int_{\boldsymbol{r}^{\prime}, 0}^{r^{\prime \prime}, N} \exp \left(-\frac{1}{l^{2}} \int_{0}^{N} \dot{\boldsymbol{r}}^{2} \mathrm{~d} \nu\right) D(\boldsymbol{r}(\nu)),
$$

where we used the notation of ref. [3] and where denotes the derivative $d / d \nu$. Next one imposes the constraint that the walk shall enclose a constant algebraic area $A$. Note that - when $r^{\prime}=r^{\prime \prime}=(0,0)$ - the area can be expressed in terms of the polymer configuration $r(\nu)=(x, y)$ as

$$
A=\frac{1}{2} \int_{0}^{N}(x \dot{y}-y \dot{x}) \mathrm{d} \nu,
$$


along with the sign convention that $A$ is counted positive (negative) if the enclosed area is located to the left (right) of the curve when traced by increasing the value of $\nu$ from 0 to $N$. Moreover, one has to take the effect of the electric field into account. Let the field $E$ be uniform with $x$ component $E_{1}$ and $y$ component $E_{2}$. Let each of the monomers have the charge $q$. The corresponding Boltzmann factor for the polymer configuration $r(\nu)$ can be expressed in the continuous form as

$$
\exp \left(q^{\prime} \int_{0}^{N} \boldsymbol{E} \cdot \boldsymbol{r} \mathrm{d} \nu\right),
$$

where $q^{\prime}=q / k_{\mathrm{B}} T, T$ being the absolute temperature of the system and $k_{\mathrm{B}}$ the Boltzmann constant. The electrostatic monomer-monomer interaction will be neglected in this paper.

Combination of (2.1) -(2.3) shows that the probability density of the area in an electric field is given by the path integral

$$
\begin{aligned}
P(A, N, E)= & \mathcal{N} \int_{r^{\prime}, 0}^{r^{\prime \prime}, N} \delta\left(A-\frac{1}{2} \int_{0}^{N}(x \dot{y}-y \dot{x}) \mathrm{d} \nu\right) \\
& \times \exp \left(-\frac{1}{l^{2}} \int_{0}^{N} \dot{r}^{2} \mathrm{~d} \nu+q^{\prime} \int_{0}^{N} E \cdot r \mathrm{~d} \nu\right),
\end{aligned}
$$

where the normalization factor $\mathcal{N}$ is determined by imposing the condition that the total probability is equal to unity. It will turn out that the thermodynamic properties of the system follow from a generating functional $Z(A, E)$ which is defined as

$$
\begin{aligned}
Z(A, E)= & \int_{r^{\prime}, 0}^{r^{\prime \prime}, N} \delta\left(A-\frac{1}{2} \int_{0}^{N}(x \dot{y}-y \dot{x}) \mathrm{d} \nu\right) \\
& \times \exp \left(\boldsymbol{\eta} \cdot \boldsymbol{r}_{1}-\frac{1}{l^{2}} \int_{0}^{N} \dot{r}^{2} \mathrm{~d} \nu+q^{\prime} \int_{0}^{N} r \cdot E \mathrm{~d} \nu\right) D(r(\nu)),
\end{aligned}
$$

where $r_{1}$ denotes the position of the monomer with label $N_{1}$ and where $\boldsymbol{\eta}=\left(\eta_{1}, \eta_{2}\right)$ is a vector introduced for convenience.

Usually one is interested in three physical quantities which can be expressed in terms of the generating functional in the following way:

(i) The configuration sum, or the probability density, $P(A, N, E)$ that the 
polymer configurations enclose an area $A$ in the presence of an electric field $\boldsymbol{E}$ is related to $Z(A, E)$ by the relation

$$
P(A, N, \boldsymbol{E})=\left.\mathcal{N} Z(A, \boldsymbol{E})\right|_{\eta=0} .
$$

(ii) The average $\left\langle\boldsymbol{r}^{2}\left(N_{1}\right)\right\rangle$ of the distance of repeating unit $N_{1}$ from the origin, squared, is given by

$$
\left\langle\boldsymbol{r}^{2}\left(N_{1}\right)\right\rangle=\left.\left(\frac{1}{Z(A, E)} \frac{\partial}{\partial \boldsymbol{\eta}} \cdot \frac{\partial}{\partial \boldsymbol{\eta}} Z(A, E)\right)\right|_{\boldsymbol{\eta}=\boldsymbol{0}} .
$$

(iii) The configuration sum density $\Omega(A, E, R)$ over all those polymer configurations which span an area equal to $A$ and for which $r\left(N_{1}\right)-r(0)=R$ has the following relation with the generating functional,

$$
\Omega(A, \boldsymbol{E}, \boldsymbol{R})=\frac{\mathcal{N}}{2 \pi} \int Z(A, \boldsymbol{E} ; \boldsymbol{\eta}=-\mathrm{i} \tau) \exp (\mathrm{i} \boldsymbol{\tau} \cdot \boldsymbol{R}) \mathrm{d} \boldsymbol{\tau} .
$$

Note that (2.6)-(2.8) give the relevant physical quantities for polymer configurations which can be open or closed. For open polymeric chains the enclosed algebraic area is defined as the area enclosed by the polymer configuration and the chord joining the endpoints. We now proceed to evaluate the generating functional.

\section{Generating functional}

In order to evaluate the generating functional $Z(A, E)$, we first represent the delta function in the integrand of $(2.5)$ by a Fourier integral. This yields

$$
Z(A, E)=\frac{1}{2 \pi} \int \tilde{Z}(g) \exp (\mathrm{i} g A) \mathrm{d} g,
$$

where $\tilde{Z}(g)$ represents the path integral

$$
\tilde{Z}(g)=\int_{r^{\prime}, 0}^{r^{\prime \prime}, N} \exp \left(-\int_{0}^{N} \mathscr{L} \mathrm{d} \nu+\boldsymbol{\eta} \cdot \boldsymbol{r}_{1}\right) D(\boldsymbol{r}(\nu))
$$

associated with a "Lagrangian" $\mathscr{L}$ which has the following explicit form:

$$
\mathscr{L}=\frac{1}{l^{2}} \dot{\boldsymbol{r}}^{2}-\frac{\lambda}{2}(x \dot{y}-y \dot{x})-q^{\prime} \boldsymbol{E} \cdot \boldsymbol{r} .
$$


Here $\lambda=-\mathrm{ig}$. It has been shown in ref. [6] that the path integral in (3.2) can be evaluated by assuming $\lambda$ to be a real number.

Next, we introduce a transformation

$$
\boldsymbol{r}=\boldsymbol{\rho}+\boldsymbol{A \nu}, \quad \boldsymbol{\rho}^{\prime}=\boldsymbol{r}^{\prime}, \quad \boldsymbol{\rho}^{\prime \prime}=\boldsymbol{r}^{\prime \prime}-\boldsymbol{A N},
$$

where $\rho$ is a two-dimensional vector with components $\xi$ and $\varphi$. Moreover, the components of the vector $A=(\alpha, \beta)$ are defined as

$$
\alpha=\frac{q^{\prime} E_{2}}{\lambda}, \quad \beta=-\frac{q^{\prime} E_{1}}{\lambda} .
$$

The transformation (3.4) reduces the path integral in (3.2) to

$$
\begin{aligned}
\tilde{Z}(g)= & Z_{1} \exp \left(\boldsymbol{\eta} \cdot \boldsymbol{A} N_{1}\right) \int_{\boldsymbol{\rho}^{\prime}, 0}^{\boldsymbol{\rho}^{\prime \prime, N}} \exp \left(-\frac{1}{l^{2}} \int_{0}^{N} \dot{\boldsymbol{\rho}}^{2} \mathrm{~d} \nu\right. \\
& \left.+\frac{\lambda}{2} \int_{0}^{N}(\xi \dot{\varphi}-\varphi \dot{\xi}) \mathrm{d} \nu+\boldsymbol{\eta} \cdot \boldsymbol{\rho}_{1}\right) D(\boldsymbol{\rho}(\nu)),
\end{aligned}
$$

where the constant $Z_{1}$ is given by

$$
\begin{aligned}
Z_{1}= & \exp \left(\frac{-2 \alpha}{l^{2}}\left(\xi^{\prime \prime}-\xi^{\prime}\right)-\frac{2 \beta}{l^{2}}\left(\varphi^{\prime \prime}-\varphi^{\prime}\right)-\frac{N}{l^{2}}\left(\alpha^{2}+\beta^{2}\right)\right) \\
& \times \exp \left[\frac{1}{2} \lambda\left(\alpha N \varphi^{\prime \prime}-\beta N \xi^{\prime \prime}\right)\right] .
\end{aligned}
$$

Since the term $\boldsymbol{\eta} \cdot \boldsymbol{\rho}_{1}$ in (3.6) depends only on the position of repeating unit $N_{1}$, it is easy to perform the path integration over all the other positions. Thus we obtain

$$
\tilde{Z}(g)=Z_{1} \exp \left(\boldsymbol{\eta} \cdot A N_{1}\right) \int G\left(\boldsymbol{\rho}^{\prime \prime}, N \mid \boldsymbol{\rho}_{1}, N_{1}\right) \mathrm{e}^{\boldsymbol{\eta} \cdot \boldsymbol{\rho}_{1}} G\left(\boldsymbol{\rho}_{1}, N_{1} \mid \boldsymbol{\rho}^{\prime}, 0\right) \mathrm{d}^{2} \boldsymbol{\rho}_{1},
$$

where the function $G\left(\boldsymbol{\rho}, N \mid \boldsymbol{\rho}_{0}, N_{0}\right)$ is defined by

$$
G\left(\boldsymbol{\rho}, N \mid \boldsymbol{\rho}_{0}, N_{0}\right)=\int_{\boldsymbol{\rho}_{0}, N_{0}}^{\rho, N} \exp \left(-\frac{1}{l^{2}} \int_{0}^{N} \dot{\boldsymbol{\rho}}^{2} \mathrm{~d} \nu+\frac{\lambda}{2} \int_{0}^{N}(\xi \dot{\varphi}-\varphi \dot{\xi}) \mathrm{d} \nu\right) D(\boldsymbol{\rho}(\nu))
$$

it can easily be shown to be related to the propagator associated with a particle 
in a magnetic field. $G$ can be evaluated in a straightforward way and is found to be given by the expression

$$
\begin{aligned}
& G\left(\xi, \varphi, N \mid \xi_{0}, \varphi_{0}, N_{1}\right)=\frac{\omega_{0}}{2 \pi l^{2} \sin \left(\frac{1}{2}\left|N-N_{1}\right| \omega_{0}\right)} \\
& \quad \times \exp \left(-\frac{\omega_{0}}{l^{2}}\left(\xi \varphi_{0}-\varphi \xi_{0}\right)\right. \\
& \left.-\frac{\omega_{0}}{2 l^{2}} \cot \left(\frac{1}{2} \omega_{0}\left|N-N_{1}\right|\right)\left[\left(\xi-\xi_{0}\right)^{2}+\left(\varphi-\varphi_{0}\right)^{2}\right]\right),
\end{aligned}
$$

where $\omega_{0}=-\frac{1}{2} \mathrm{i} g l^{2}$.

Substituting the last formula into (3.8) and performing the integration over $\rho_{1}$ we arrive at the following expression for $\tilde{Z}(g)$ :

$$
\begin{aligned}
\tilde{Z}(g)= & \frac{\omega_{0}}{2 \pi l^{2} \sin \left(\frac{1}{2} N \omega_{0}\right)} Z_{1} \exp \left[\boldsymbol{\eta} \cdot\left(\boldsymbol{\rho}^{\prime}+\boldsymbol{A} N_{1}\right)\right] \\
& \times \exp \left(-\frac{\omega_{0}}{l^{2}}\left(\xi^{\prime} \varphi^{\prime \prime}-\varphi^{\prime} \xi^{\prime \prime}\right)\right) \\
& \times \exp \left(-\frac{\omega_{0}}{2 l^{2}}\left(\delta^{2}+\psi^{2}\right) \cot \left[\frac{1}{2}\left(N-N_{1}\right) \omega_{0}\right]\right) \\
& \times \exp \left\{\frac{\omega_{0}}{2 l^{2}} \frac{\sin \left[\frac{1}{2}\left(N-N_{1}\right) \omega_{0}\right] \sin \left(\frac{1}{2} N_{1} \omega_{0}\right)}{\sin \left(\frac{1}{2} N \omega_{0}\right)}\right. \\
& \times\left[\left(\psi+\frac{\eta_{1} l^{2}}{\omega_{0}}+\delta \cot \left[\frac{1}{2}\left(N-N_{1}\right) \omega_{0}\right]\right)^{2}\right. \\
& \left.\left.+\left(\psi \cot \left[\frac{1}{2}\left(N-N_{1}\right) \omega_{0}\right]+\frac{\eta_{2} l^{2}}{\omega_{0}}-\delta\right)^{2}\right]\right\},
\end{aligned}
$$

where for brevity we have set $\delta=\xi^{\prime \prime}-\xi^{\prime}$ and $\psi=\varphi^{\prime \prime}-\varphi^{\prime}$. This expression for $\tilde{Z}(g)$, when inserted into (3.1), yields the generating functional.

\section{Closed polymer loops}

To evaluate the statistical mechanical properties of closed polymer loops we must obtain the generating functional for the closed configurations. For this purpose we should set $\boldsymbol{r}^{\prime \prime}=\boldsymbol{r}^{\prime}$ in the expression (3.11), which by virtue of transformation (3.4) implies that $\xi^{\prime \prime}-\xi^{\prime}=-\alpha N$ and $\varphi^{\prime \prime}-\varphi^{\prime}=-\beta N$. Also we set $\xi^{\prime}=\varphi^{\prime}=0$, which only results in a shift of the origin of coordinates. With these substitutions the Fourier transform $\tilde{Z}(g)$ of the generating functional, 
which we now denote as $\tilde{Z}_{c}(g)$, becomes

$$
\begin{aligned}
\tilde{Z}_{\mathrm{c}}(g)= & \frac{\theta}{\pi N l^{2} \sinh \theta} \exp \left(-\frac{a E^{2}}{\theta^{2}}(1-\theta \operatorname{coth} \theta)\right) \\
& \times \exp \left(p \chi^{2}\right) \exp \left[b \chi_{1}\left(\frac{\mathrm{i} \sigma E_{2}}{\theta}+p\left\{E_{1}-\mathrm{i} E_{2} \operatorname{coth}[(1-\sigma) \theta]\right\}\right)\right] \\
& \times \exp \left[b \chi_{2}\left(-\frac{\mathrm{i} \sigma E_{1}}{\theta}+p\left\{E_{2}+\mathrm{i} E_{1} \operatorname{coth}[(1-\sigma) \theta]\right\}\right)\right]
\end{aligned}
$$

where

$$
\begin{aligned}
& \theta=N g l^{2} / 4, \quad \sigma=N_{1} / N, \\
& p=\frac{\sinh [(1-\sigma) \theta] \sinh (\sigma \theta)}{\theta \sinh \theta}, \\
& a=\frac{1}{16} N l^{2} Q^{2}, \quad b=\frac{1}{2} Q \sqrt{N l^{2}}, \quad Q=N q^{\prime}, \\
& \chi^{2}=\frac{1}{4} N l^{2} \eta^{2} .
\end{aligned}
$$

Once again, we can substitute this expression of $\tilde{Z}_{c}(g)$ in (3.1) to obtain the generating functional for closed polymer loops, which will be denoted by $Z_{\mathrm{c}}(A)$. Having obtained the expression for the generating functional we can now investigate the statistical mechanical properties.

\subsection{The probability distribution}

To obtain the probability distribution for the area enclosed by a polymer loop we set $\boldsymbol{\eta}=\boldsymbol{O}$ in (4.1) and use the defining relation (2.6) to write

$$
\begin{aligned}
P_{\mathrm{c}}(A, N, E)= & \frac{2}{\pi N l^{2}} \exp \left(\frac{a E^{2}}{3}\right) \int_{-\infty}^{\infty} \frac{\theta}{\sinh \theta} \\
& \times \exp \left(-\frac{a E^{2}}{\theta^{2}}(1-\theta \operatorname{coth} \theta)+\frac{4 \mathrm{i} \theta A}{N l^{2}}\right) \mathrm{d} \theta .
\end{aligned}
$$

This expression agrees with the result obtained earlier in ref. [11]; it has been analysed in detail there. In particular, it has been shown that in a strong electric field $P_{\mathrm{c}}(A, N, E)$ behaves as

$$
\left.P_{\mathrm{c}}(A, N, E)\right|_{\eta=0} \simeq\left(\frac{5 \pi}{a}\right)^{1 / 2} \frac{18}{\pi N l^{2} E} \exp \left(-\frac{180 A^{2}}{\left(N l^{2}\right)^{2} a E^{2}}\right) \text {. }
$$




\subsection{Evaluation of $\left\langle\boldsymbol{r}^{2}\left(N_{1}\right)\right\rangle$}

As has been stated earlier, the mean square distance $\left\langle r^{2}\left(N_{1}\right)\right\rangle$ is defined by (2.7). It is easy to see that for closed polymer loops

$$
\begin{aligned}
\frac{\partial}{\partial \boldsymbol{\eta}} \cdot \frac{\partial}{\partial \boldsymbol{\eta}} \tilde{Z}_{\mathrm{c}}(g)= & \left(\frac{N l^{2}}{4}\right)\left[4 p+4 a E^{2}\left(\frac{2 \sigma p \operatorname{coth}[(1-\sigma) \theta]}{\theta}-\frac{\sigma^{2}}{\theta^{2}}\right.\right. \\
& \left.\left.-\frac{p^{2}}{\sinh ^{2}[(1-\sigma) \theta]}\right)\right],
\end{aligned}
$$

hence the expression for the mean square distance takes the form

$$
\begin{aligned}
\left\langle\boldsymbol{r}^{2}\right\rangle= & Z_{\mathrm{c}}^{-1}(A, E) \frac{N l^{2}}{4} \int_{-\infty}^{\infty} \frac{\theta}{\left(\pi N l^{2}\right) \sinh \theta} \\
& \times\left[4 p+4 a E^{2}\left(\frac{2 \sigma p \operatorname{coth}[(1-\sigma) \theta]}{\theta}-\frac{\sigma^{2}}{\theta^{2}}-\frac{p^{2}}{\sinh ^{2}[(1-\sigma) \theta]}\right)\right] \\
& \times \exp \left(-\frac{a E^{2}}{\theta^{2}}(1-\theta \operatorname{coth} \theta)\right) \exp \left(\frac{4 \mathrm{i} \theta A}{N l^{2}}\right) \mathrm{d} \theta
\end{aligned}
$$

The analytical behaviour of $\left\langle r^{2}\left(N_{1}\right)\right\rangle$ described by (4.6) is quite complicated. However, it is easy to verify that as $E \rightarrow 0$ we recover the expression for the mean square distance derived earlier in [9]. Moreover, we can analyze the behaviour of $\left\langle\boldsymbol{r}^{2}\left(N_{1}\right)\right\rangle$ when the electric field is very strong. For this purpose we consider the integral

$$
I=\int \frac{\theta}{\sinh \theta}\left[N p l^{2}+4 a E^{2} f(\theta)\right] \exp \left(-a E^{2} g(\theta)+\frac{4 \mathrm{i} \theta A}{N l^{2}}\right) \mathrm{d} \theta,
$$

where for convenience we have set

$$
\begin{aligned}
& f(\theta)=\frac{2 \sigma p \operatorname{coth}[(1-\sigma) \theta]}{\theta}-\frac{\sigma^{2}}{\theta^{2}}-\frac{p^{2}}{\sinh ^{2}[(1-\sigma) \theta]}, \\
& g(\theta)=\frac{1-\theta \operatorname{coth} \theta}{\theta^{2}} .
\end{aligned}
$$

To use the methods of asymptotic analysis we rewrite the integrand $F(\theta)$ in (4.7) as

$$
F(\theta)=\frac{\theta}{\sinh \theta}\left(N p l^{2}-\frac{4 a f(\theta)}{g(\theta)} \frac{\partial}{\partial a}\right) \exp \left(-a E^{2} g(\theta)+\frac{4 \mathrm{i} \theta \lambda}{N l^{2}}\right) .
$$


Now we observe that the saddlepoint of $g(\theta)$ is at $\theta=0$. Therefore, the integral in (4.7) can be approximated by

$$
I \simeq\left(N p(0) l^{2}-\frac{4 a f(0)}{g(0)} \frac{\partial}{\partial a}\right) \int \exp \left(-\frac{a E^{2} \theta^{2}}{45}+\frac{4 \mathrm{i} \theta A}{4 l^{2}}\right) \mathrm{d} \theta,
$$

which can be immediately evaluated and written as

$$
I \simeq\left[N p(0) l^{2}-\frac{4 a f(0)}{g(0)}\left(-\frac{1}{2 a}+\frac{180 A^{2}}{\left(N l^{2}\right)^{2} a^{2} E^{2}}\right)\right] I_{1},
$$

where the quantity $I_{1}$ is given by

$$
I_{1}=\sqrt{\frac{45 \pi}{a E^{2}}} \exp \left(-\frac{180 A^{2}}{\left(N l^{2}\right)^{2} a E^{2}}\right) .
$$

Following identical arguments, one can show that for large electric fields the inverse of the generating functional $Z^{-1}(A, E)$ can be approximated by $I_{1}$. Hence we have

$$
\left\langle r^{2}\left(N_{1}\right)\right\rangle \simeq N p(0) l^{2}-\frac{4 a f(0)}{g(0)}\left(-\frac{1}{2 a}+\frac{180 A^{2}}{\left(N l^{2}\right)^{2} a^{2} E^{2}}\right) .
$$

Substituting explicitly the value of various quantities into (4.14) we obtain

$$
\left\langle r^{2}\left(N_{1}\right)\right\rangle=\left[N_{1}(1-\sigma) l^{2}+12 a \sigma^{2}(1-\sigma)^{2}\left(-\frac{1}{2 a}+\frac{180 A^{2}}{\left(N l^{2}\right)^{2} a^{2} E^{2}}\right)\right],
$$

which is the behaviour of $\left\langle\boldsymbol{r}^{2}\left(N_{1}\right)\right\rangle$ for large electric fields. It shows that in the presence of an electric field the mean square distance behaves as $A^{2}$, which is a typical sign of topological rigidity. This rigidity is superimposed on the normal behavior $\left\langle\boldsymbol{r}^{2}\right\rangle \sim N_{1}$, as indicated by the first term of (4.15).

\subsection{The entropic force}

In order to evaluate the entropic force on the repeating unit $N_{1}$ we substitute $\boldsymbol{\eta}=-\mathrm{i} \boldsymbol{\xi} \cdot \boldsymbol{R}$ and evaluate the integral in (2.8) to arrive at the following expression for the configuration sum density $\Omega(A, E, R)$ :

$$
\begin{aligned}
\Omega(A, \boldsymbol{E}, \boldsymbol{R})= & \frac{4}{\left(N l^{2}\right)^{2}} \int_{-\infty}^{\infty} \frac{\theta}{p(\theta) \sinh (\theta)} \exp \left(-a E^{2} g(\theta)-\frac{a E^{2}}{p(\theta)} f(\theta)\right. \\
& \left.-\frac{R^{2}}{N l^{2} p(\theta)}+\frac{Q R_{1}}{2 p(\theta)}+\frac{Q R_{2}}{2 p(\theta)}\right) \\
& \times \cos \left(\left(R_{1} E_{2}-R_{2} E_{1}\right) h(\theta)+\frac{4 A \theta}{N l^{2}}\right) \mathrm{d} \theta,
\end{aligned}
$$


where the function $h(\theta)$ has the form

$$
h(\theta)=\frac{\sigma}{\theta}-p(\theta) \operatorname{coth}[(1-\sigma) \theta]
$$

The entropic force on the repeating unit $N_{1}$ is related to the configuration sum density $\Omega$ by the relation

$$
f(A, R)=\frac{k_{\mathrm{B}} T}{\Omega} \nabla \Omega
$$

and can be calculated easily from (4.17). However, this expression is too difficult to analyze analytically. It will be clear anyhow that the configuration sum density is a highly non-linear function of $R$, which suggests that the entropic force on the repeating unit would be a highly non-linear function of $R$. This is an indication of the dramatic effects of the area constraint.

\section{Open chains}

To study the statistical mechanical properties of open chains we set $\xi^{\prime}=$ $\varphi^{\prime}=0$ in (3.11) and integrate over $\xi^{\prime \prime}, \varphi^{\prime \prime}$. This yields the Fourier transform of the generating functional, which we denote by $\tilde{Z}_{\mathrm{o}}(g)$. Similarly we denote the generating functional associated with the open configurations by $Z_{\mathrm{o}}(A)$. The explicit expression for $\tilde{Z}_{\mathrm{o}}(g)$ is

$$
\begin{aligned}
\tilde{Z}_{\mathrm{o}}(g)= & \frac{1}{\cosh \theta} \exp \left[a E^{2}\left(\frac{1}{\theta^{2}}-\frac{\tanh \theta}{\theta^{3}}+\frac{\tanh \theta}{\theta}\right)\right] \\
& \times \exp \left(\chi^{2} p_{\mathrm{o}}\right) \exp \left[b \chi _ { 1 } \left(\frac{\mathrm{i} \sigma E_{2}}{\theta}+\frac{p_{\mathrm{o}}^{\prime}}{\theta^{2}}\left\{E_{1}-\mathrm{i} E_{2} \operatorname{coth}[(1-\sigma) \theta]\right\}\right.\right. \\
& \left.\left.+\frac{p_{\mathrm{o}}^{\prime}}{\theta}\left\{E_{1} \operatorname{coth}[(1-\sigma) \theta]-\mathrm{i} E_{2}\right\}\right)\right] \\
& \times \exp \left[b \chi _ { 2 } \left(-\frac{\mathrm{i} \sigma E_{1}}{\theta}+\frac{p_{\mathrm{o}}^{\prime}}{\theta^{2}}\left\{E_{2}+\mathrm{i} E_{1} \operatorname{coth}[(1-\sigma) \theta]\right\}\right.\right. \\
& \left.\left.+\frac{p_{\mathrm{o}}^{\prime}}{\theta}\left\{E_{2} \operatorname{coth}[(1-\sigma) \theta]+\mathrm{i} E_{1}\right\}\right)\right]
\end{aligned}
$$

where

$$
\begin{aligned}
& p_{o}=\frac{\sinh \theta \cosh [(1-\sigma) \theta]}{\theta \cosh \theta}, \\
& p_{o}^{\prime}=\frac{\sinh (\sigma \theta) \sinh [(1-\sigma) \theta]}{\cosh \theta} .
\end{aligned}
$$


Once we have the expression for $\tilde{Z}_{\mathrm{o}}(g)$ we can in an analogous way proceed to investigate the thermodynamic properties.

\subsection{The probability distribution}

We set $\boldsymbol{\eta}=0$ in eq. (5.1) and use the defining relation (2.6) to arrive at the probability density of what is known in mathematics as "Levy's area", which is associated with open configurations enclosing a fixed area. This expression reads as

$$
\begin{aligned}
P_{\mathrm{o}}(A, N, E)= & \frac{\exp \left(-\frac{2}{3} a E^{2}\right)}{\pi N l^{2}} \int \frac{1}{\cosh \theta} \exp \left(\frac{4 \mathrm{i} \theta A}{N l^{2}}\right) \\
& \times \exp \left[a E^{2}\left(\frac{1}{\theta^{2}}-\frac{\tanh \theta}{\theta^{3}}+\frac{\tanh \theta}{\theta}\right)\right] \mathrm{d} \theta
\end{aligned}
$$

The area distribution defined by the last equation is quite complicated. However, for strong electric fields (which is the region of interest) one can apply the techniques of asymptotic analysis. It is easy to see that the integrand contributes most in the neighborhood of the point $\theta=0$. In this range we can approximate

$$
\frac{\tanh \theta}{\theta}+\frac{1}{\theta^{2}}-\frac{\tanh \theta}{\theta^{3}} \simeq \frac{2}{3}-\frac{7}{15} \theta^{2},
$$

while $\cosh \theta$ can be taken to be unity. In this case the integral over $\theta$ can be easily performed and one finds

$$
P_{\mathrm{o}}(A, N, E) \simeq \frac{1}{\pi N l^{2} E} \sqrt{\frac{15 \pi}{7 a}} \exp \left(-\frac{60}{7\left(N l^{2}\right)^{2} E^{2} a}\right) .
$$

This shows that, although the actual analytic behaviour of the distribution functions for the open polymeric chains (5.3) and close polymer loops (4.4.) is quite different, both of them behave in a Gaussian way (of course with a different spread) in strong electric fields. This is perhaps due to the fact that in strong electric fields all the monomers tend to align themselves in the direction of the field. Hence the polymer takes the shape of a "needle", irrespective of the fact whether the chain is open or closed.

\subsection{The mean square distance}

The evaluation of mean square distance $\left\langle r^{2}\left(N_{1}\right)\right\rangle$ proceeds in a way which is similar to the calculation performed in section 4.2 , for closed polymer loops. Onc finds 


$$
\left\langle\boldsymbol{r}^{2}\left(N_{1}\right)\right\rangle=Z_{\mathrm{o}}^{-1}(A, E) I_{\mathrm{o}},
$$

where $I_{\mathrm{o}}$ is given by the integral

$$
I_{\mathrm{o}}=\int_{-\infty}^{\infty} \frac{\theta}{\cosh \theta}\left[4 p_{\mathrm{o}}(\theta)+b^{2} E^{2} f_{\mathrm{o}}(\theta)\right] \exp \left(a E^{2} g_{\mathrm{o}}(\theta)+\frac{4 \mathrm{i} \theta A}{N l^{2}}\right) \mathrm{d} \theta,
$$

where for the open chains the functions $f_{0}(\theta)$ and $g_{0}(\theta)$ have the form

$$
\begin{aligned}
f_{\mathrm{o}}(\theta)= & \frac{2 p_{\mathrm{o}}^{\prime}(\theta) \sigma E^{2}}{\theta^{3} \tanh [(1-\sigma) \theta]}-\frac{p_{\mathrm{o}}^{\prime 2}}{\theta^{4} \sinh ^{2}[(1-\sigma) \theta]} \\
& -\frac{\sigma^{2}}{\theta^{2}}+\frac{p_{\mathrm{o}}^{\prime 2}}{\theta^{2} \sinh ^{2} \theta}+\frac{2 \sigma p_{\mathrm{o}}^{\prime}}{\theta^{2}}, \\
g_{\mathrm{o}}(\theta)= & \frac{1}{\theta^{2}}-\frac{\tanh \theta}{\theta^{3}}+\frac{\tanh \theta}{\theta} .
\end{aligned}
$$

We can again use asymptotic analysis to investigate the behaviour of $\left\langle\boldsymbol{R}^{2}\left(N_{1}\right)\right\rangle$ for strong electric fields, in the present case of open chains. Such a calculation yields

$$
\left\langle r^{2}\left(N_{1}\right)\right\rangle \simeq \frac{N l^{2}}{4}\left[4 p_{\mathrm{o}}(0)+\frac{b^{2} f_{\mathrm{o}}(0)}{g_{\mathrm{o}}(0)}\left(-\frac{1}{2 a}+\frac{60 A^{2}}{7 a^{2}\left(N l^{2}\right)^{2} E^{2}}\right)\right] .
$$

One can calculate the values of $f_{\mathrm{o}}(0)$ and $g_{\mathrm{o}}(0)$ from (5.8) and (5.9), respectively. However, it is obvious from the last equation that $\left\langle\boldsymbol{r}^{2}\left(N_{1}\right)\right\rangle$ behaves as $A^{2}$ which, as has been stated earlier, is a signature of rigidity. Eq. (5.10) also indicates that the analytical behaviour of the mean square distance $\left\langle\boldsymbol{r}^{2}\left(N_{1}\right)\right\rangle$ for both closed loops and open chains is similar for the case of strong electrical fields. This is expected in view of the similarity in the behaviour of the respective probability distributions.

\section{The asymptotic evaluation}

In most situations of practical interest exact results are not available. Hence, it is desirable to develop schemes for an approximate evaluation of the constrained path integrals, and to compare them with the exact results.

The simplest problem which is amenable to exact analysis corresponds to the case of non-interacting planar polymer loops constrained to enclose a fixed area. The exact distribution of the area was derived in an earlier paper by 
Khandekar and Wiegel [6]. Hence, we now study a semiclassical evaluation of the path integral corresponding to this case for comparison.

\subsection{Free case}

The Lagrangian $\mathscr{L}$ for a non-interacting plane polymer can be written as

$$
\mathscr{L}=\dot{\boldsymbol{r}}^{2} / l^{2} \text {. }
$$

We introduce the constraint that the polymer loops enclose a fixed area $A$ by using the Lagrange multiplier $\lambda$ and modifying the Lagrangian of (6.1) to read

$$
\mathscr{L}=\frac{\dot{\boldsymbol{r}}^{2}}{l^{2}}-\frac{\lambda}{2}(x \dot{y}-\dot{y} x-A) .
$$

The Euler-Lagrange equations for the problem are given by

$$
\begin{aligned}
& \ddot{x}+\omega \dot{y}=0, \\
& \ddot{y}-\omega \dot{x}=0,
\end{aligned}
$$

where $\omega=\lambda l^{2} / 2$. These equations can be readily solved to yield

$$
\begin{aligned}
& x=A \cos (\omega \nu)-B \cos [\omega(N-\nu)]-C_{2} / \omega, \\
& y=A \sin (\omega \nu)+B \sin [\omega(N-\nu)]+C_{1} / \omega
\end{aligned}
$$

$A, B, C_{1}$ and $C_{2}$ are arbitrary constants to be determined by the boundary conditions on the optimal trajectory, i.e. $x(0)=x^{\prime}, x(N)=x^{\prime \prime} ; y(0)=y^{\prime}$ and $y(N)=y^{\prime \prime}$. This leads to:

$$
\begin{aligned}
& A=\frac{1}{2}\left(\frac{X}{\cos (\omega N)-1}+\frac{Y}{\sin (\omega N)}\right), \\
& B=\frac{1}{2}\left(\frac{X}{\cos (\omega N)-1}-\frac{Y}{\sin (\omega N)}\right), \\
& C_{1}=\omega\left[y^{\prime \prime}-A \sin (\omega N)\right], \\
& C_{2}=\omega\left[A \cos (\omega N)-B-x^{\prime \prime}\right] .
\end{aligned}
$$

Using these values of the constants it is easy to see that the value of the action 
functional $S=\int_{0}^{N} \dot{r}^{2} / l^{2} \mathrm{~d} \nu$ along the optimal trajectory is given by

$$
S=\frac{\omega^{2} N}{l^{2}}\left(X^{2}+Y^{2}\right) \frac{1}{2[1-\cos (\omega N)]},
$$

with $\omega$ yet to be determined. Once $\omega$ is determined, the configuration sum $G\left(x^{\prime \prime}, y^{\prime \prime}, N \mid x^{\prime}, y^{\prime}, 0\right)$ can be approximated as

$$
G\left(x^{\prime \prime}, y^{\prime \prime}, N \mid x^{\prime}, y^{\prime}, 0\right)=\sum \operatorname{det}\left|\frac{\partial^{2} S}{\partial r^{\prime \prime} \partial r^{\prime}}\right| \mathrm{e}^{-s},
$$

where the sum is to be carried out over all permissible values of $\omega$. The value of $\omega$ can be determined by demanding

$$
A=\frac{1}{2} \int_{0}^{N}(x \dot{y}-y \dot{x}) \mathrm{d} \nu,
$$

which leads to the equation

$$
\begin{aligned}
A & =\frac{1}{2} \omega\left[l^{2} S-\left(X C_{1}+Y C_{2}\right)\right] \\
& =\frac{l^{2} S}{2 \omega}-\frac{1}{2}\left[x^{\prime \prime} y^{\prime}-y^{\prime \prime} x^{\prime}+\frac{1}{2} \cot (\omega N / 2)\left(X^{2}+Y^{2}\right)\right] .
\end{aligned}
$$

Next, let us concentrate on closed polymer chains; this implies $x^{\prime \prime} \rightarrow x^{\prime}$ and $y^{\prime \prime} \rightarrow y^{\prime}$. Then it is clear from eqs. (6.4) that $\omega= \pm 2 n \pi, n=1,2, \ldots$ Moreover, the normalizability of the configuration sum imposes the condition that $\omega>0(<0)$ if $A>0(<0)$. The values of $\omega= \pm 2 n \pi$ correspond to the singular points of $S$ as well as of $A$. Hence, in order to take the appropriate limit we set $\omega=2 n \pi+\delta ; \delta \rightarrow 0$ in eq. (4.8b). This yields

$$
A=n \pi\left(\frac{X^{2}+Y^{2}}{\delta^{2}}\right)+\mathcal{O}(\delta)
$$

and

$$
C_{1} X+C_{2} Y=\omega\left(x^{\prime \prime} y^{\prime}-y^{\prime \prime} x^{\prime}\right)+\mathcal{O}(\delta) .
$$

Substituting the value of $C_{1} X+C_{2} Y$ from (6.10) into (6.8b) it is easy to see that

$$
\operatorname{det}\left|\frac{\partial^{2} S}{\partial^{2} r^{\prime \prime} \partial^{2} r^{\prime}}\right|=\frac{\omega}{l^{2}}=\left|\frac{2 n \pi}{l^{2} N}\right| .
$$


It is also straightforward to show that the points $\omega_{n}=2 n \pi$ correspond to singularities (caustics) of $S$. Therefore, as we cross the singularity the propagator is multiplied by a phase factor. Thus, in this approximation, the propagator is given by

$$
G\left(x^{\prime \prime}, y^{\prime \prime}, N \mid x^{\prime}, y^{\prime}, 0\right)=\frac{1}{\pi} \sum_{n=1}^{\infty}\left(\mathrm{e}^{\mathrm{i} \pi / 2}\right)^{2(n-1)} \frac{2 n \pi}{l^{2} N} \mathrm{e}^{-S_{n}},
$$

where $S_{n}$ can be evaluated using eq. (6.8b) by setting $x^{\prime \prime}=x^{\prime}$ and $y^{\prime \prime}=y^{\prime}$. This yields the complete expression for the configuration sum as:

$$
\begin{aligned}
G\left(x^{\prime \prime}, y^{\prime \prime}, N \mid x^{\prime}, y^{\prime}, 0\right) & =\sum_{n=1}^{\infty} \mathrm{e}^{\mathrm{i} \pi(n-1)} \frac{n}{l^{2} N} \exp \left(-\frac{4 n \pi A}{l^{2} N}\right) \\
& =\left[2 N l^{2} \cosh ^{2}\left(2 \pi A / N l^{2}\right)\right]^{-1},
\end{aligned}
$$

which is the exact result derived earlier by Khandekar and Wiegel [6].

A few remarks are necessary at this point. First, it is clear from eq. (6.13) that the first term of the series represents the leading contribution to the configuration sum, provided $A / l^{2} N \gg 1$. Moreover, as long as the Lagrangian $\mathscr{L}$ is quadratic, one can recover the exact expression for the configuration sum by considering the contributions from all the classical trajectories, and incorporating the appropriate phase factors arising from the singularities.

Having demonstrated that the optimal trajectory leads to the dominant contribution to the configuration sum, we now proceed to consider noninteracting polymers in an electric field.

\subsection{Polymer loops in an electric field}

We proceed as in section 6.1. The Lagrangian $\mathscr{L}$ now reads

$$
\mathscr{L}=\frac{\dot{x}^{2}+\dot{y}^{2}}{l^{2}}-q\left(E_{1} x+E_{2} y\right)-\frac{\lambda}{2}(x \dot{y}-y \dot{x}-A),
$$

where $q$ is a charge on each monomer; $E_{1}$ and $E_{2}$ refer to the $x$ and $y$ components of the electric field $\boldsymbol{E}$ respectively; $\lambda$ is the Lagrange multiplier. As before, the Euler-Lagrange equations are given by

$$
\begin{aligned}
& \frac{2 \ddot{x}}{l^{2}}+\lambda \dot{y}+q E_{1}=0, \\
& \frac{2 \ddot{y}}{l^{2}}-\lambda \dot{x}+q E_{2}=0,
\end{aligned}
$$


subject to the condition $x(0)=x(N)=0, y(0)=y(N)=0$.

We now introduce a transformation

$$
\begin{array}{ll}
\xi=x-\alpha \nu, & \eta=y-\beta \nu, \\
\alpha=-q E_{1} / \lambda, & \beta=q E_{2} / \lambda .
\end{array}
$$

The set of equations (6.15) simplifies to read

$$
\ddot{\xi}+\omega \dot{\eta}=0, \quad \ddot{\eta}-\omega \dot{\xi}=0
$$

which can be readily solved to yield

$$
\begin{aligned}
& \xi=A \cos (\omega \nu)-B \cos [(N-\nu) \omega]-C_{2} / \omega \\
& \eta=A \sin (\omega \nu)+B \sin [(N-\nu) \omega]+C_{1} / \omega
\end{aligned}
$$

where the constants $A, B, C_{1}$ and $C_{2}$ can be determined by using the boundary conditions on the trajectory. This gives

$$
\begin{aligned}
& A=\frac{N}{2}\left(\frac{\alpha}{1-\cos (\omega N)}-\frac{\beta}{\sin (\omega N)}\right), \\
& B=\frac{N}{2}\left(\frac{\alpha}{1-\cos (\omega N)}+\frac{\beta}{\sin (\omega N)}\right), \\
& \frac{C_{1}}{\omega}=-B \sin (\omega N)=-\frac{N}{2}\left(\frac{\alpha \sin (\omega N)}{1-\cos (\omega N)}+\beta\right), \\
& \frac{C_{2}}{\omega}=\frac{(A-B)[(1+\cos (\omega N)]+\alpha N}{2}=\frac{N}{2}\left(\alpha-\frac{1+\cos (\omega N)}{\sin (\omega N)}\right) .
\end{aligned}
$$

With these values of the constants we should now evaluate the action functional $S$,

$$
S=\int_{0}^{N} \frac{\left(\dot{x}^{2}+\dot{y}^{2}\right)}{l^{2}} \mathrm{~d} \nu-q \int_{0}^{N}\left(E_{1} x+E_{2} y\right) \mathrm{d} \nu .
$$

Towards this end one observes that

$$
\int_{0}^{N} \frac{\left(\dot{x}^{2}+\dot{y}^{2}\right)}{l^{2}} \mathrm{~d} \nu=\frac{1}{l^{2}}\left(\frac{\omega^{2} N^{3}}{2[(1-\cos (\omega N)]}\left(\alpha^{2}+\beta^{2}\right)-N\left(\alpha^{2}+\beta^{2}\right)\right)
$$


and

$$
q \int_{0}^{N}\left(E_{1} x+E_{2} y\right) \mathrm{d} \nu=\frac{q^{2} l^{2} E^{2}}{2 \omega}\left(\frac{N}{\omega}-\frac{N^{2} \cot (\omega N / 2)}{2}\right) ;
$$

hence the value of $S$ is given by

$$
S=\frac{q^{2} l^{2} E^{2}}{2 \omega^{3}}\left(\frac{x^{3}}{\sin ^{2} x}-3 x+2 x^{2} \cot x\right),
$$

where $x$ is a scaled variable related to $\omega$ by $x=\omega T / 2$. As before, the value of $\omega$, and hence of $x$, is to be determined by demanding

$$
A=\frac{1}{2} \int_{0}^{N}(x \dot{y}-y \dot{x}) \mathrm{d} \nu,
$$

which implies

$$
2 A=\frac{q^{2} E^{2} l^{4}}{2 \omega^{3}}\left(\frac{x^{2}}{\sin ^{2} x}+x \cot x-2\right) .
$$

Thus we observe that in the case of entangled polymers in an electric field, $x$ is determined by a transcendental equation. Therefore, further calculations can not be performed analytically. However, in order to determine the smallest root one can use the approximation $\sin x \simeq x-\frac{1}{6} x^{3}$. In that case one recovers the configuration sum in the asymptotic limit. This agrees with the expression for the configuration sum which was derived by us in ref. [11].

\section{Conclusions}

In this paper we considered non-interacting planar polymer configurations entangling with uniformly distributed singularities and subject to an external electric field. The effect of entanglement was simulated by imposing a constraint which compels the polymer configurations to enclose a fixed algebraic area. Whereas the term "area" is only meaningful for closed curves, it was generalized to include open polymer configurations. For open configurations we define the enclosed area as the area enclosed by the closed curve formed by the polymer configuration and the straight line joining the end-points of the polymer.

Within the framework of this model exact expressions for the configuration 
sums corresponding to both open and closed chains were obtained. The generating functional was used to find an expression for the mean square end-to-end distance. In the last section we developed an approximation technique for the evaluation of the configuration sum. It was shown that the results are accurate in the asymptotic regime $A / N l^{2} \gg 1$.

\section{Acknowledgements}

D.C. Khandekar would like to acknowledge the support and hospitality of the Department of Applied Physics at Twente University, during the autumn of 1991 , which made this collaboration possible. He would also like to thank the Bhabha Atomic Research Centre for granting him a leave of absence.

\section{References}

[1] S.F. Edwards, Proc. Phys. Soc. 91 (1967) 513.

[2] D.C. Khandekar and F.W. Wiegel, Phys. Lett. A 127 (1988) 379.

[3] F.W. Wiegel, Introduction to Path-Integral Methods in Physics and Polymer Science (World Scientific, Singapore, New Jersey, 1986).

[4] D.C. Khandekar and S.V. Lawande, Phys. Rep. 137 (1986) 115.

[5] M.G. Brereton and C. Butler, J. Phys. London A 20 (1987) 3955.

[6] D.C. Khandekar and F.W. Wiegel, J. Phys. London A 21 (1988) L563.

[7] P. Levy, Am. J. Math. 62 (1940) 487.

[8] B. Duplantier, J. Phys. London A 22 (1989) 3033.

[9] D.C. Khandekar and F.W. Wiegel, J. Stat. Phys. 53 (1988) 1073.

[10] D.C. Khandekar and F.W. Wiegel, J. Phys. (Paris) 50 (1989) 263.

[11] F.W. Wiegel and D.C. Khandekar, Int. J. Mod. Phys. B 6 (1992) 3147. 